International Journal of Distributed and Parallel Systems (IJDPS) Vol.3, No.4, July 2012

\title{
Performance of Power Decentralized DETECTION IN WIRELESS SENSOR SYSTEM WITH DS-CDMA
}

\author{
Ali M. Fadhil ${ }^{1}$, Haider M. AlSabbagh ${ }^{2}$, and Turki Y. Abdallah ${ }^{1}$ \\ ${ }^{1}$ Department of Computer Engineering, College of Engineering, University of Basra, \\ Basra, Iraq \\ ali_muayed@yahoo.com, protryounis@yahoo.com \\ ${ }^{2}$ Department of Electrical Engineering, College of Engineering, University of Basra, \\ Basra, Iraq \\ haidermaw@ieee.org
}

\begin{abstract}
Wireless sensor networks take great importance in recent years according to their potential applications in different areas like health monitoring, military applications, tactile system and industrial applications. In this paper the decentralized sensing with noise and band limited channel between the sensor nodes and merging stations (fusion center) for different levels of power is analyzed. The evolution of the system performance is based on the considering the wireless sensor network with direct sequence-code division multiple access (DS-CDMA) for varying levels of power. The achieved results indicate the performance is improved with employing the direct sequence-code division multiple accesses (DS-CDMA). In the situation of large sensor systems and random spreading, the decentralized detection execution is derived supposition independent and identically distributed sensor observation via random matrix theory.
\end{abstract}

\section{KEYWORDS}

DECENTRALIZED DETECTION, WIRELESS SENSOR SYSTEM, DS-CDMA, FUSION CENTER.

\section{1-INTRODUCTION}

Wireless Sensor Networks (WSNs) used in different applications such as enhanced manufacturing productivity, evolving emergency state, and health monitoring [1]. The wireless sensor network consists of hundreds to thousands of sensors nodes distributed in specific areas, and the server connects with a number of sensors through radio links. Data is composed at the wireless node \& send to the server straightforward or to another sensor and then to the server. The date is presented by the server connectors [2,3]. The intelligent environment specifies the coming growth stage in constriction, tools, and industrial. To found data from real world sensing information done by different sensors distributed in specific areas, the challenge in step is finding out the relevant amount, for combination of data, estimate the data. The date needed by intelligent environment is done by wireless sensor network [4]. The characteristics of wireless sensor networks contain cooperative signal processing involving data querying from the end sensors and data fusion from multiple sensors [5]. The energy source provided for sensor nodes in wireless sensor networks is usually battery and operated on a demand applications, cannot reaching the level that the sensor nodes operate in long time without 
International Journal of Distributed and Parallel Systems (IJDPS) Vol.3, No.4, July 2012

recharging, so the power consumption represented the most challenges in wireless sensor network [6].

The distributed detection system consists of number of information, linked to a fusion center, which implemented by Wireless sensor networks. The decentralized detection system performs significant benefits as a centralized system; according to data gathering and data fusion are implemented at the same node In wireless sensor networks the sensors node communicate between each other or with the data gathering node that performs detection and fusion. These nodes used the shared communication channel in normal condition for interchange of data between each other. This process needed high communication bandwidth and energy consumption between sensor nodes and fusion center, the decentralized sensing and information merging problem produce for two decades $[7,8]$. There is quantity prior work of the decentralized detection which ignores the influence of the noisy channels between the local sensors and information merging center. The one of the major important in wireless sensor networks is to expand the full network lifetime when the power is specific [9]. In [10] the researchers presented two new linear receiver structures for synchronous non-orthogonal DSCDMA wireless sensor network. Propos non-orthogonal communication between sensors and a data fusion center via DS-CDMA and investigate the fusion performance in the presence of channel error due to both multiple-access interference (MAI) and noise in [11]. And For a wireless sensor network (WSN) with a random number of sensors, perform a decision fusion rule that uses the total number of detection reported by local sensors as a statistic for hypothesis testing [12].

In this paper we analyze the performance of power with probability of fusion error for the decentralized sensing with noise and band limited channels with large wireless sensor networks. The bandwidth limited is got into account by supposition non-orthogonal directsequence code-division multiple-access (DS-CDMA) communication between sensors and information merging station. The spread spectrum techniques have performed for wireless sensor networks, the CDMA is a promising multiple access scheme for wireless sensor networks due to its interference averaging properties $[13,14]$.

The rest of the paper is arranged as follows; section 2 presents the system model. In section 3 the achieved result and analyses are given. Then, the main conclusions are summarized.

\section{2-THE SYSTEM MODEL DESCRIPTION}

Consider a binary hypothesis experiment problem in an Ns-node wireless sensor network linked to the information fusion station. Express the null \& alternative hypothesis by $\left(\mathrm{K}_{0}\right)$ and $\left(\mathrm{K}_{1}\right)$, continually, taking the probabilities $\mathrm{P}\left(\mathrm{K}_{0}\right)=\mathrm{P}_{0}$ and $\mathrm{P}\left(\mathrm{K}_{1}\right)=\mathrm{P}_{1}$. Under the two hypothesis the $\mathrm{n}$ th local sensor noticing $z_{n}$ for $\mathrm{n}=1, \ldots . \mathrm{N}_{\mathrm{s}}$, can be written as

$$
\begin{aligned}
& \mathrm{K}_{0}: z_{n}=X_{0, n}+v_{n} \\
& \mathrm{~K}_{1}: z_{n}=X_{1, n}+v_{n}
\end{aligned}
$$

The two Gaussian signals of interest, denoted by $X_{0, n}$ and $X_{1, n}$, where the noticing noise $v_{n}$ is assumed to be zero-mean Gaussian with the collection of noise samples having a conversance matrix $\Gamma_{\mathrm{v}}$.the local decision $v_{n}\left(z_{n}\right)$ which are sent to the fusion center ,denote by $\mathrm{r}\left(u_{1}\left(\mathrm{z}_{1}\right), u_{2}\right.$ $\left.\left(\mathrm{z}_{2}\right), \ldots \ldots \ldots, u_{N s}\left(z_{N s}\right)\right)$ the received signal at the fusion center. The fusion center makes a final decision according to decision rule $u_{0}(r)$. The problem at hand is to choose $u_{0}(r), u_{1}\left(z_{1}\right), u_{2}$ 
$\left(\mathrm{z}_{2}\right) \ldots u_{N S}\left(z_{N S}\right)$ so that a chosen performance metric is optimized, Before retransmission to the fusion center [13], the local sensor decision sent to the fusion center are given by

$$
u_{n}=\mathrm{G} z_{n} \quad \text { for } \mathrm{n}=1, \ldots N_{s}
$$

Where $\mathrm{G}>0$ is the analog relay amplifier gain at each node, all sensor nodes share a common bandwidth and a total available energy in this model. For analytical reasons, as well as due to their practical relation to DS-CDMA communications, and the bandwidth sharing nonorthogonal Communication based on spreading in which each sensor node is assigned a signature code of length $N$. if the n-th sensor node is assigned the code $s_{n}$, the received chipmatched filtered and sampled discrete-time signal at the fusion center can be written as

$$
\mathbf{r}=\mathrm{G} \sum_{i=1}^{N S} S_{\mathrm{n}} Z_{n}+\mathbf{w}=\mathrm{g} \boldsymbol{S}_{\mathbf{z}}+\mathbf{w}
$$

where $\mathbf{r}$ and $\mathbf{w}$ are $N$-dimensional received signal and receiver noise vectors, continually and the $\mathrm{n}$-th Colum of $\mathrm{N} \times \mathrm{N}_{\mathrm{s}}$ matrix $\mathrm{S}$ is equal to the vector $s_{n}$, the receiver noise is a white Gaussian noise process so that the filtered noise vector $\mathrm{w} \sim \mathrm{N}\left(0, \sigma^{2}{ }_{\mathrm{w}} \mathrm{I}_{\mathrm{N}}\right)$, then

$$
\begin{aligned}
& \mathrm{K}_{0:} \mathrm{r} \sim \mathrm{N}\left(\mathrm{m}_{0}, \Gamma_{0}\right) \\
& \mathrm{K}_{1:} \mathrm{r} \sim \mathrm{N}\left(\mathrm{m}_{1}, \Gamma_{1}\right)
\end{aligned}
$$

For $\mathrm{j}=0,1$

$$
\begin{aligned}
& m_{j}=\mathrm{G} S E\left\{X_{j}\right\} \\
& \Gamma_{\mathrm{j}}=\mathrm{G}^{2} \mathrm{~S}\left(\operatorname{Cov}\left(X_{j}\right)+\Gamma_{\mathrm{v}}\right) S^{T}+\sigma_{W}^{2} I_{N} .
\end{aligned}
$$

Consider the detection of a deterministic signal so that $\mathrm{X}_{1}=-\mathrm{X}_{0}=\mathrm{m} 1$ is known $(\mathrm{m}>0)$ and $\quad \Gamma$ ${ }_{0}=\Gamma_{1}=\Gamma$ where $(1$ is the vector of all ones)

$$
\Gamma=\mathrm{G}^{2} \mathrm{~S} \Gamma_{\mathrm{v}} S^{T}+\sigma_{W}^{2} I_{N}
$$

With these assumptions, from (5), and

$$
\mathrm{m}_{1}=-\mathrm{m}=\mathrm{GmS} 1
$$

The radiated power of node $\mathrm{n}$ is then given by

$$
E\left\{\left|u_{n}\right|^{2}\right\}=G^{2} E\left\{\left|z_{n}\right|^{2}\right\}=G^{2}\left(m^{2}+\sigma_{v}^{2}\right) \text {. }
$$

Where $\sigma_{v}^{2}$ is the observation noise variance, Let us define the total power the whole system is submitted to as $P$, and the amplifier gain $(G)$ is related to the size of the sensor system and total available power $\mathrm{P}$ as 


$$
\mathrm{G}=\sqrt{\frac{P}{\mathrm{~N}_{\mathrm{s}}\left(m^{2}+\sigma_{\mathrm{v}}^{2}\right)}}
$$

Then, it can be shown that the optimal threshold rule at the fusion center is of the form

$$
u_{0}(\mathrm{r})= \begin{cases}1, & T(r) \geq \tau^{\prime} \\ 0, & T(r)<\tau^{\prime}\end{cases}
$$

Where defined the decision variable $\mathrm{T}$ as

$$
\begin{aligned}
T(r) & =\left(m_{1}-m_{0}\right)^{T} \Gamma^{-1} r \\
& =2 G m 1^{T} S^{T}\left(G^{2} S \Gamma_{v} S^{T}+\sigma_{w}^{2} I_{N}\right)^{-1} r
\end{aligned}
$$

And $\tau^{\prime}$ is the threshold that depends on the specific optimality criteria. The false-alarm $\mathrm{P}_{\mathrm{f}}$ and Miss $\mathrm{P}_{\mathrm{m}}$ probabilities are given by

$$
\mathrm{P}_{\mathrm{f}}=\mathrm{Q}\left(\frac{\tau^{\prime}+2 G^{2} m^{2} 1^{T} S^{T} \Gamma^{-1} S^{-1} S^{2} S 1}{2 G m \sqrt{1^{T} S^{T} \Gamma^{-1} S 1}}\right)
$$

And

$$
\mathrm{P}_{\mathrm{m}}=\mathrm{Q}\left(\frac{2 G^{2} m^{2} 1^{T} S^{T} \Gamma^{-1} S^{-1} S^{2} S 1-\tau^{\prime}}{2 G m \sqrt{1^{T} S^{T} \Gamma^{-1} S 1}}\right)
$$

In large sensor system performance analysis, the spreading code $s_{n}$ are chosen randomly so that each element of $s_{n}$ takes either $\frac{1}{\sqrt{N}}$ or $-\frac{1}{\sqrt{N}}$ with equal probability, moreover, take independent sensor observation such that $\Gamma_{\mathrm{v}}=\sigma^{2}{ }_{\mathrm{v}} \mathrm{I}$. Let us assume a large sensor system such that both $\mathrm{N}_{\mathrm{S}}$ and $\mathrm{N}$ are large such that $\lim _{N \rightarrow \infty} \frac{N_{S}}{N}=\alpha$.

Now using a theorem on the convergence of the empirical distribution of eigenvalues of a large random matrix proven in [15].

Where $\beta^{*}$ is the unique positive solution to the fixed point equation.

$$
\beta^{*}=\left[\sigma^{2}+\alpha \int \frac{p}{1+p \beta^{*}} d F(p)\right]^{-1}
$$

Using this theorem we may prove the following proposition, with $\mathrm{S}$ and $\Gamma$ define as above,

$$
\mathrm{G}^{2} 1^{\mathrm{T}} \mathrm{S}^{\mathrm{T}} \Gamma^{-1} \mathrm{~S} 1 \rightarrow\left(\frac{\sigma_{v}^{2}}{N_{S}}+\frac{m^{2}+\sigma_{v}^{2}}{P \beta_{0}}\right)^{-1}
$$


Almost surely, as $\mathrm{N} \rightarrow \infty$, where

$$
\beta_{0}=\frac{\sqrt{\left(\gamma+\sigma_{\mathrm{w}}^{2}\right)^{2} \alpha^{2}+2 \gamma\left(\sigma_{\mathrm{w}}^{2}-\gamma\right) \alpha+\gamma^{2}-\left(\gamma+\sigma_{\mathrm{w}}^{2}\right) \alpha+\gamma}}{2 \gamma \sigma^{2}}
$$

With $\gamma=\frac{P}{N\left(1+\frac{m^{2}}{\sigma_{W}^{2}}\right)}$ and $\Gamma_{\mathrm{v}}=\sigma_{\mathrm{v}}^{2} \mathrm{I}$.

using the definitions of $\mathrm{S}$ and 1 , led to

$$
\mathrm{G}^{2} 1^{\mathrm{T}} \mathrm{S}^{\mathrm{T}} \Gamma^{-1} \mathrm{~S} 1=\mathrm{G}^{2}\left(\sum_{n=1}^{N s} S_{n}^{T} \Gamma^{-1} S_{n}+\sum_{\substack { N=1 \\
\begin{subarray}{c}{n^{\prime}=1 \\
n^{\prime} \neq n{ N = 1 \\
\begin{subarray} { c } { n ^ { \prime } = 1 \\
n ^ { \prime } \neq n } }\end{subarray}}^{N s} S_{n}^{T} \Gamma^{-1} S_{n^{\prime}}\right)
$$

Let $l$ denotes asset of sensor indices (i.e. $\left.l \in\left\{1,2, \ldots, N_{S}\right\}\right), S_{A}$ denote the matrix $S$ with column indices specified by set $A$ denoted, $\Lambda_{n}=G^{2} \sigma^{2}{ }_{v} I_{n}$ and $Q_{A}=\left(S_{A} \Lambda_{N s}-|A| S_{A}+\sigma^{2}{ }_{w} I_{N}\right)$ where $\mathrm{I}_{\mathrm{n}}$ and $\mid \mathrm{Al}$ are the $n \times n$ identity matrix and the cardinality of set $\mathrm{A}$, respectively. Then, for $n=1 \ldots N_{S}$, using the matrix inversion lemma we have that

$$
\begin{aligned}
S_{n}^{T} \Gamma^{-1} s_{n} & =s_{n}^{T}\left(\mathrm{G}^{2} \sigma^{2}{ }_{\mathrm{v}} s_{n} s_{n}^{T}+\mathrm{S}_{\{\mathrm{n}\}} \Lambda_{\mathrm{Ns}-1} \mathrm{~S}_{\{\mathrm{n}\}}+\sigma^{2}{ }_{\mathrm{w}} \mathrm{I}_{\mathrm{N}}\right)^{-1} s_{n} \\
& =\frac{s_{n}^{T} Q_{\{n\}}^{-1} s_{n}}{1+G^{2} \sigma_{\mathrm{v}}^{2} s_{n}^{T} Q_{\{n\}}^{-1} s_{n}}
\end{aligned}
$$

But, applying theorem 1, we can show that

$$
S_{n}^{T} \mathrm{Q}_{\{\mathrm{n}\}^{-1}} S_{n} \rightarrow \beta_{0}
$$

Almost surely, where $\beta_{0}$ is as given by (13) with $\gamma=\frac{P}{N\left(1+\frac{m^{2}}{\sigma^{2} v}\right)}$. Substituting (16) in (15) we have almost surely.

$$
S_{n}^{T} \Gamma^{-1} S_{n} \rightarrow\left(\frac{1}{\beta_{0}}+\mathrm{G}^{2} \sigma_{v}^{2}\right)^{-1}
$$

Similarly, reported application of matrix inversion lemma twice followed by the use of theorem 1 show that, for $n \neq n$ '

$$
S_{n}^{T} \Gamma^{-1} S_{n}=\frac{s_{n}^{T} Q_{\left\{n, n^{\prime}\right\}}^{-1} S_{n^{\prime}}}{\left(1+G^{2} \sigma_{v}^{2} S_{n}^{T} Q_{\{n\}}^{-1} S_{n}\right)\left(1+G^{2} \sigma_{v}^{2} S_{n}^{T} Q_{\left\{n, n^{\prime}\right\}}^{-1} S_{n \prime}\right)} \rightarrow 0
$$


The proposition leads to the following corollary on the asymptotically large sensor system performance of decentralized detection in noisy band limited channels, the large sensor network performance of the decentralized detection is given by

$\mathrm{Pf} \rightarrow \mathrm{Q}\left(\frac{\tau^{\prime}+2 m^{2}\left(\frac{\sigma_{\mathrm{V}}^{2}}{N s}+\frac{m^{2}+\sigma_{\mathrm{V}}^{2}}{P \beta 0}\right)}{2 m\left(\frac{\sigma_{\mathrm{V}}^{2}}{N s}+\frac{m^{2}+\sigma_{\mathrm{V}}^{2}}{P \beta 0}\right)^{-1 / 2}}\right)^{-1}$

And

$\mathrm{Pm} \rightarrow \mathrm{Q}\left(\frac{2 m^{2}\left(\frac{\sigma^{2} v}{N s}+\frac{m^{2}+\sigma^{2} v}{P \beta 0}\right)^{-1}-\tau \prime}{2 m\left(\frac{\sigma^{2} v}{N s}+\frac{m^{2}+\sigma^{2} v}{P \beta 0}\right)^{-1 / 2}}\right)$

The above corollary leads to insight on large sensor system performance of decentralized detection in noisy, band limited channels as well as to important system design considerations. Consider the special case of minimum probability of error optimality design at the fusion center which leads to $\tau^{\prime}=0$. Then according to corollary 1 , the large system probability of error is asymptotically given by

$$
\begin{aligned}
P_{e}(\sigma) & =\mathrm{Q}\left(\mathrm{mG} \sqrt{1^{T} S^{T} \Gamma^{-1} S 1}\right) \text { where } \lambda=\frac{\sigma_{v}^{2}}{N_{s}}+\frac{m^{2}+\sigma_{v}^{2}}{P \beta_{0}} \\
& \rightarrow \mathrm{Q}(m / \sqrt{\lambda})
\end{aligned}
$$

Where convergence is almost surely, $N_{S}=$ number of sensors.

In fact for large alpha, one can show that $\beta_{0}=1 / \sigma_{\mathrm{w}}^{2}$, and as a result, in this case the error probability in (20) goes to where $q_{o}=\frac{P}{\sigma_{w}^{2}}, q_{c}=\frac{\sigma_{v}^{2}}{m^{2}}[15,16]$.

$P_{e}(\alpha) \rightarrow \mathrm{Q}\left(\sqrt{\frac{q_{o}}{\left(1+q_{c}\right)}}\right)$

Where $\sigma_{w}^{2}$ is the receiver noise power at the fusion center, on the other hand, if one were to allocate all available power $\mathrm{P}$ and the total bandwidth to just one sensor node the fusion center performance will be given by

$$
P_{e, 1}=\mathrm{Q}\left(\sqrt{\frac{q_{o}}{\frac{q_{O}}{1 / q_{c}}+\left(1+q_{c}\right)}}\right)
$$


International Journal of Distributed and Parallel Systems (IJDPS) Vol.3, No.4, July 2012

\section{3-THE RESUlts AND ANALYSES}

Figure 1 shows the relationship between the fusion probability of error and the level of power in Eq.20, in large sensor system $\left(N_{S}=N \times \alpha\right), N$ is the large spreading lengths. We find the fusion error probability decrease when the available power increases; to improve the system the available power which may permit the minimum level of fusion probability error should be provided to improve the system performance. Then, the same amount of power to the specific value of probability of error which turn to improve the system performance.



Figure 1: large sensor system to the level of power, $\alpha=0.1 \& 0.2, q_{c}=4$.

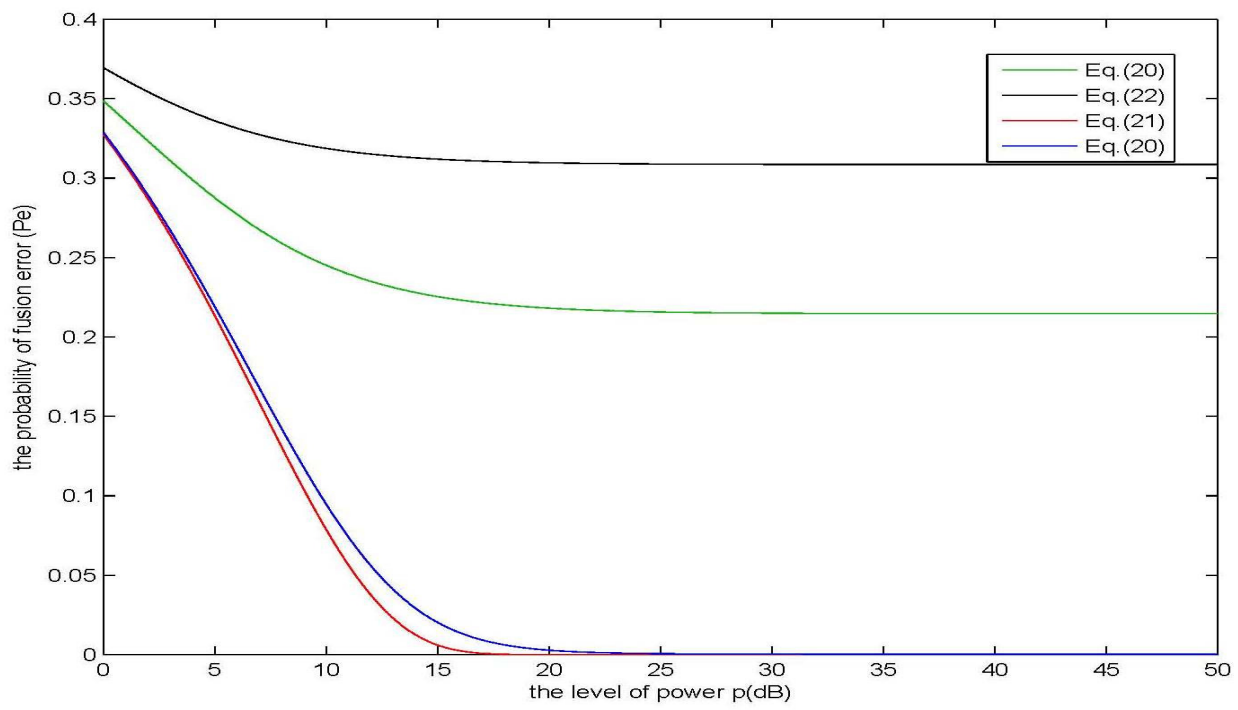

Figure 2 . The large sensor system to the deferent level of power when $q_{c}=4$, $\mathrm{a}=0.1 \mathrm{~N}=25,500$. 
Figure 2 illustrates effect of probability of fusion error to different value of power to three equation and find the relation, in Eq.20 find the probability of error decrease when the power increase which led to increase the system performance but with increasing in power when using different value of spreading length $(\mathrm{N}=5,500)$. In Eq. 21 for large alpha the probability is decreasing with specific level to the fusion error probability. In Eq.22 we use one sensor node and the probability of error is higher than in that in Eqs. 20 \& 21 in level of errors. This led to save power by using a local decision for different sensor replacing specific power for one sensor node with band limited channel, which leading to improving the system performance.

\section{4-CONCLUSIONS}

The paper presents the decentralized detection analysis for different levels of power for wireless sensor network with band limited channels and noise, the system based on using the DS-CDMA communication between the local sensors and merging station. the derived a closed form expression for the merging analysis, consider large sensor network with random spreading and use of random matrix theory to get the detection execution that is autonomous of the selecting spreading codes. Used the system with noise and band limited it useful to merge many sensors local decisions as possible batter than one sensor to save power of the system, by calculate the fusion probability of error to different value of power for the system with three equation. This leads to save power by using a local decision for different sensor replacing specific power for one sensor node with band limited channel.

\section{REFERENCE}

[1] J. Zheng. \&A. Jamalipour, (2009) Wireless Sensor Networks: A network perspective, Wiley, USA.

[2] C. Buratti, A. Conti, D. Dardari , \& R. Verdone (2009) “ An Overview on Wireless Sensor Networks Technology and Evolution”, Sensors, Vol.9,pp 6869-6896.

[3] A. M. Khedr, \& H. Ramadan (2011), “Effective Sensor Relocation Technique in Mobile Sensor Networks" International Journal of Computer Networks \& Communications (IJCNC), Vol.3, No.1.

[4] N. Trigoni, \& B. Krishnamachari (2012), "Sensor network algorithms and applications", Phil. Trans. R. Soc. A 370,pp 5-10.

[5] R. Niu, P. K. Varshney, \& Q. Cheng (2006) "Distributed detection in a large wireless sensor network", Elsevier Information Fusion, Vol. 7, pp 380-394.

[6] S.Tarannum (2010) "Energy Conservation Challenges in Wireless Sensor Networks: A Comprehensive Study" Wireless Sensor Network, Vol.2, pp 483-491.

[7] Saeed A. Aldosari. \& José M. F. Moura,( 2004) "Detection in Decentralized Sensor Networks", IEEE Journal on Selected Areas in Communication. Vol.2, pp. 277-280.

[8] Ke Liu. \& Akbar M. Sayeed ( 2007) “Type-Based Decentralized Detection in Wireless Sensor Networks", IEEE Transactions on signal processing, Vol. 55, No. 5. pp 1899 - 1910.

[9] A. Appadwedula. V.V. Veeravalli. \& D. L. Jones,( 2005) "Energy-Efficient Detection in Sensor Networks”, IEEE Journal on Selected Areas in Communication, Vol. 23, No. 4, pp 693 - 702.

[10] J. S. Dyer. \& B. Natarajan, (2007) "Improved Low-Complexity Fusion Receivers for Synchronous DS-CDMA Sensor Networks", IEEE Journal on Selected Areas in Communication, pp.11231126.

[11] S. K Jayaweera, (2005) "Optimal Bayesian Data Fusion and Low-complexity Approximations for Distributed DS-CDMA Wireless Sensor Networks in Rayleigh Fading”, IEEE Proc,pp.19-24. 
International Journal of Distributed and Parallel Systems (IJDPS) Vol.3, No.4, July 2012

[12] R. Niu. \& P. K. Varshney, (2005) "Distributed Detection and Fusion in a Large Wireless Sensor Network of Random Size”, EURASIP Journal on Wireless Communications and Networking Vol.4, pp 462-472.

[13] R. X. Gao. \&P. Hünerberg,(2002) "Design of a CDMA-Based Wireless Data Transmitter For Embedded Sensing”, IEEE Transaction on Instrumentation and Measurement, Vol. 51, No. 6.

[14] M. D. Moghadam, H. Bakhshi, \& G. Dadashzadeh (2011) “DS-CDMA Cellular Systems Performance with Base Station Assignment, Power Control Error, and Beamforming over Multipath Fading" International Journal of Computer Networks \& Communications(IJCNC), Vol.3, No.1.

[15] J. Chamberland and V. V. Veeravalli, (2004) "Asymptotic results for decentralized detection in power constrained wireless sensor networks", IEEE Journal on Select. Areas in Commun., Vol. 22, No. 6, pp. 1007 - 1015.

[16] J. Evans and D. N. C. Tse,(2000) "Large System Performance of Linear Multiuser Receivers In Multipath Fading Channels”, IEEE Trans. Inform. Theory, vol. 46, pp. 2059-2078. 